Document downloaded from:

http://hdl.handle.net/10251/60353

This paper must be cited as:

Ortigosa, N.; Cano, O.; Ayala Gallego, G.; Galbis Verdu, A.; Fernandez Rosell, C. (2014). Atrial fibrillation subtypes classification using the General Fourier-family Transform. Medical Engineering and Physics. 36(4):554-560. doi:10.1016/j.medengphy.2013.12.005.

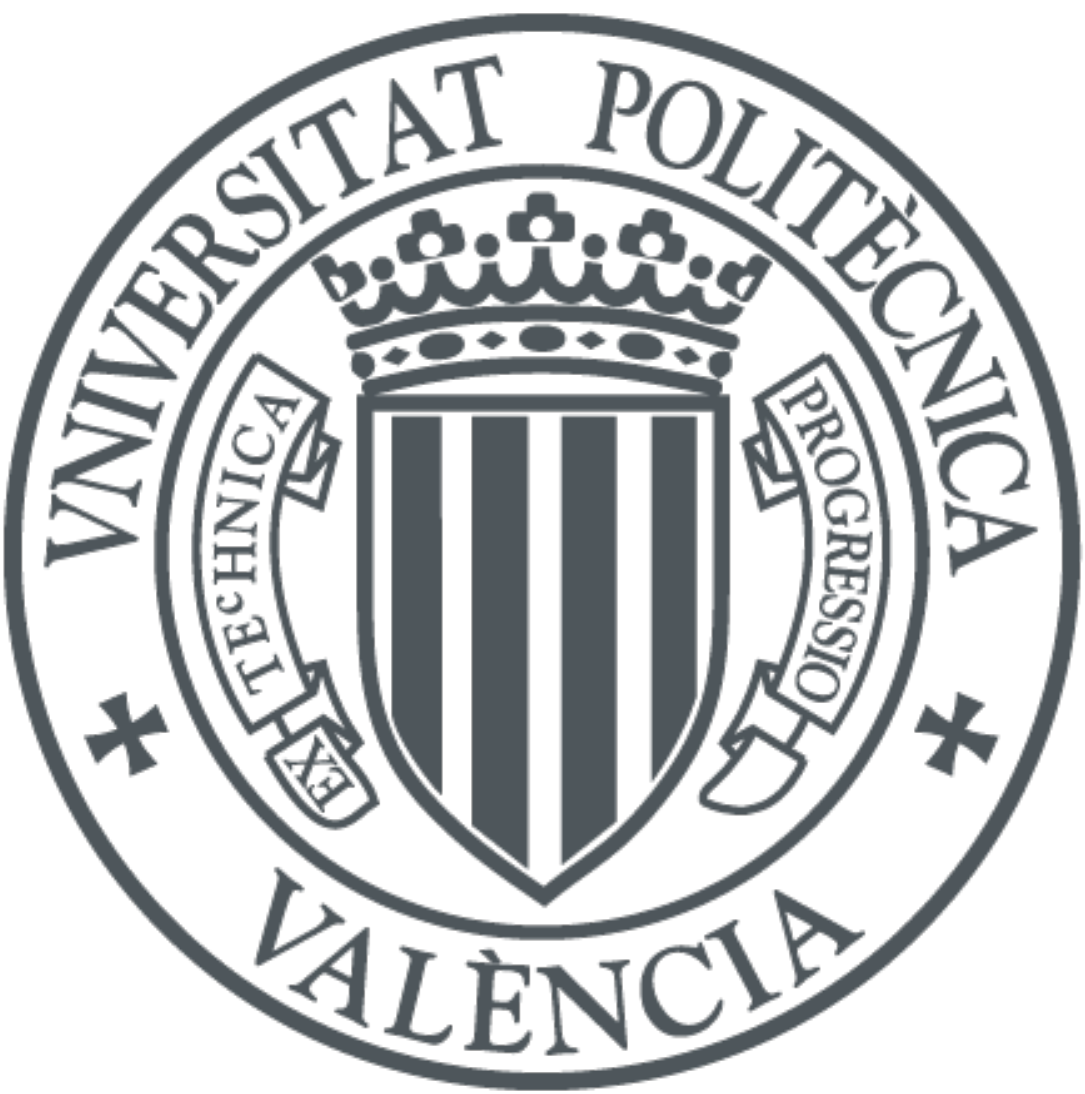

The final publication is available at

http://dx.doi.org/10.1016/j.medengphy.2013.12.005

Copyright Elsevier

Additional Information 


\section{Atrial fibrillation subtypes classification using the General Fourier-family Transform}

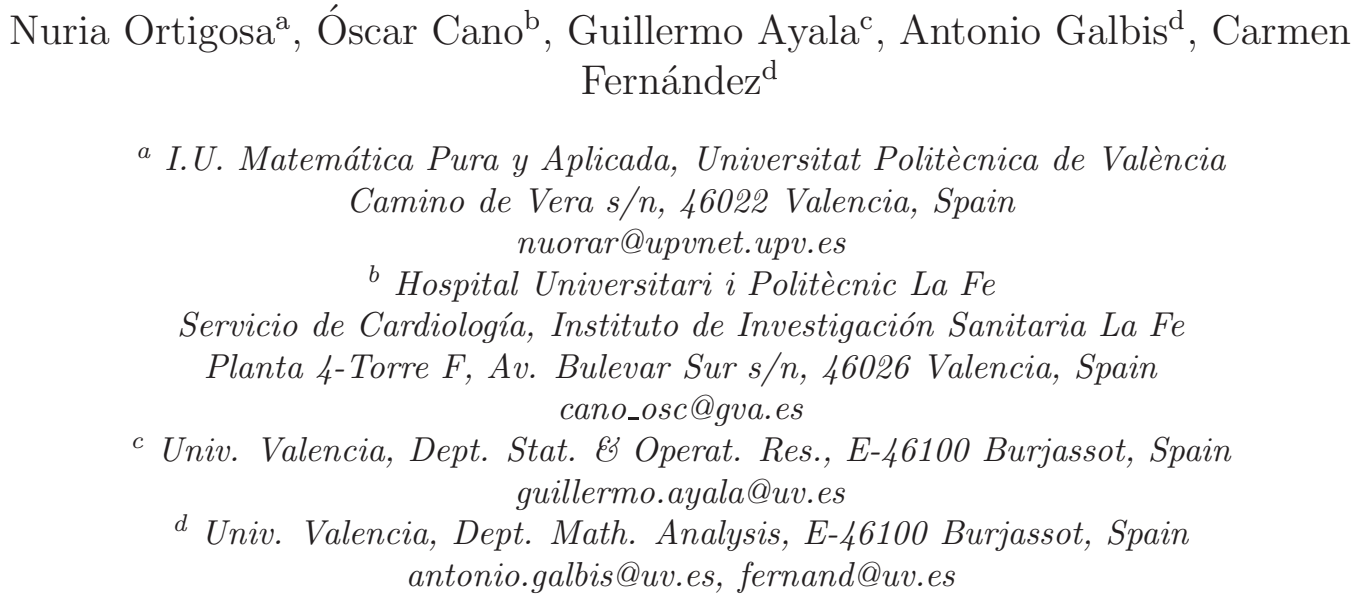

\section{Abstract}

Atrial fibrillation patients can be classified into paroxysmal, persistent and permanent attending to the temporal pattern of this arrhythmia. The surface electrocardiogram hides this differentiation. A classification method to discriminate between the different subtypes of atrial fibrillation by using short segments of electrocardiograms recordings is presented. We will process the electrocardiograms (ECGs) using time-frequency techniques with a global accuracy of $80 \%$. Real cases are evaluated showing promising results for an implementation in a semiautomated diagnostic system.

Keywords: Atrial fibrillation, Time-frequency transforms, S-transform, Generalized Fourier Transform 


\section{Introduction}

Atrial fibrillation (AF) is the most common sustained arrhythmia in clinical practice with a global prevalence of $1-2 \%$. This prevalence of AF increases with age up to $5-15 \%$ for people of 80 years old ${ }^{1}$.

Atrial fibrillation is characterized by a very rapid, chaotic rhythm in which atria and ventricles are unsynchronized. This arrhythmia is produced by a continuous reentry of an electrical impulse in the atria ${ }^{2}$. This is reflected on the electrocardiogram (ECG) by absence of the $\mathrm{P}$ waves and the presence of an undulating baseline. Based on the temporal pattern of this arrhythmia, patients are usually classified as paroxysmal, persistent and permanent ${ }^{3}$. AF episodes may be paroxysmal if they terminate spontaneously, usually within seven days, or persistent if the patient requires pharmacological or electrical cardioversion to restore sinus rhythm ${ }^{4}$. Permanent atrial fibrillation is de-

fined when rhythm control interventions are not pursued and the presence of the arrhythmia is accepted by the patient and the physician.

Research on atrial fibrillation detection and analysis using the surface electrocardiogram signal has been specially extensive during last decade ${ }^{5}$. References which analyse AF in ECGs usually make use of temporal or Fourier-based spectral techniques in order to characterize the fibrillation process $^{6}$. For example, Martínez et al. $(2012)^{7}$ assessed the risk of suffering from an atrial fibrillation episode by studying the P-wave features and their evolution, whereas Kotska et al. $(2011)^{8}$ and Bukkapatnam et al. (2008) ${ }^{9}$ use wavelets and non-linear time analysis series to extract features to detect and 
classify AF episodes, respectively.

In this manner, the Physionet/Computers in Cardiology Challenge of 2004 proposed to predict the spontaneous termination of atrial fibrillation, and provided three different groups of one-minute ECG records ${ }^{10}$ : nonterminating, soon-terminating, or terminating $\mathrm{AF}$, depending on whether AF terminates at least for one hour, one minute or one second after the end of the record, respectively. Hence, many classification methods have been presented using the above public database.

For instance, Nilsson et al. (2006) ${ }^{11}$ analysed fibrillatory frequency and the exponential decay of the harmonics depending on the terminating or non-terminating AF, whereas Sandberg et al. (2008) ${ }^{12}$ tracked the dominant frequency in atrial fibrillation episodes to improve accuracy in the arrhythmia analysis. In addition, Alcaraz et al. $(2009,2010)^{13,14}$ predicted the spontaneous termination of AF by studying the use of sample entropy of atrial activity organization prior to paroxysmal atrial fibrillation in contrast to the quantification of the recurrence plot combined with a multilayer perceptron neural network that Sun et al. (2008) presented ${ }^{15}$. Moreover, Valenzuela et al. $(2009)^{16}$ proposed to use as most features as possible with satisfactory results for other references to classify AF using genetic programming.

Thus, the majority of the works that address the challenge proposed by Physionet in 2004 are mainly focused in the analysis of frequency changes to predict termination of AF episodes (generally frequency changes abruptly for spontaneous termination in contrast to gradually decaying of drug-induced 
termination ${ }^{17}$ ). Nevertheless, recent references point out that although good classification results can be obtained by classifying the records of the database provided by Physionet, it is still necessary to propose a method that consistently performs well across various and different scenarios ${ }^{18}$.

So, in this paper we propose a method to classify subtypes of atrial fibrillation (paroxysmal and persistent) by means of the feature extraction from the General Fourier-family time-frequency transform and a Support Vector Machine classification. One value-add of this work is the population sample: Subjects in our study belong to a heterogeneous group, since there are first episode and recurrent paroxysmal segments, different antiarrhythmic drugs and recurrent AF episodes after catheter ablation.

\section{Materials}

Consecutive unselected patients with paroxysmal or persistent atrial fibrillation who were treated in a specific arrhythmia clinic of a tertiary center conformed the study population. Subtypes of AF were defined according to current guidelines $^{3,1}$. Thus, a patient was considered to have paroxysmal AF if the episode was self-terminated usually within 7 days. Persistent AF was considered if the episode lasted longer than 7 days or required termination either with drugs or with electrical cardioversion. Clinical management of the patients was left at the discretion of the attending cardiologist. Nonpharmacological treatments included electrical cardioversion and pulmonary vein isolation (either transvenous or surgically guided). 
A total of 71 atrial fibrillation signals were included in the study, 56 signals corresponded to patients with persistent atrial fibrillation and 15 signals were obtained from patients with paroxysmal atrial fibrillation. ECG signals in lead II were acquired at sampling rate of $500 \mathrm{~Hz}$ and an amplitude resolution of $5 \mu \mathrm{V}$ over an amplitude range of $\pm 5 \mathrm{mV}$. Duration of analyzed signals was 5 seconds. Baseline characteristics of the population sample are described in Table 1. Patients with persistent atrial fibrillation were older, had larger left atrium diameters and were treated more frequently with electric cardioversion and ACE inhibitors when compared with paroxysmal atrial fibrillation patients.

\section{Methods}

\subsection{Time-frequency transforms}

The Fourier Transform is probably the most important signal analysis tool, since it provides the frequency spectrum with globally referenced phase measurements. Nevertheless, it is not able to provide information about how the signal frequency content varies along time. This is a drawback in the study of biomedical signals, as frequency content variations are often of paramount importance in order to perform a thorough analysis.

The Short-Time Fourier Transform (STFT) introduces information about frequency changes in spectral response with respect to time. This information is obtained by means of dividing the signal into fragments and multiplying each one by a window (often a Gaussian). Then, the Fourier Transform is 
Table 1: Statistical summaries of our database. Hypertension was defined as a systolic blood pressure $\geq 140 \mathrm{~mm} \mathrm{Hg}$, a diastolic blood pressure $\geq 90 \mathrm{~mm} \mathrm{Hg}$, or if the patient was prescribed antihypertensive medication(s). Diabetes mellitus was defined as serum fasting glucose $\geq 7.0 \mathrm{mmol} / \mathrm{L}$ or on medications. Hypercolesterolemia was defined as cholesterol $\geq 6.4 \mathrm{mmol} / \mathrm{L}$ or treatment with lipid-lowering drugs. Structural heart disease is defined as LV hypertrophy $>15 \mathrm{~mm}, L V E F<50 \%$, moderate or greater degrees of valvulopathy, prior myocardial infarction, significant coronary artery disease or the presence of primary myocardial diseases. AF: Atrial fibrillation. ACE: angiotensin converter enzyme. ARBs: angiotensin receptor blockers. LV: left ventricle.

\begin{tabular}{lrrrr}
\hline & $\begin{array}{r}\text { Paroxysmal } \\
\text { AF (n=15) }\end{array}$ & $\begin{array}{r}\text { Persistent } \\
\text { AF }(\mathrm{n}=56)\end{array}$ & $\begin{array}{r}\text { Overall } \\
(\mathrm{n}=71)\end{array}$ & P value \\
\hline Age (mean, range) & $52(28-83)$ & $63(39-86)$ & $61(28-86)$ & 0.017 \\
Male (n, \%) & $10(67 \%)$ & $37(66 \%)$ & $47(66 \%)$ & 1 \\
Hypertension (n, \%) & $7(47 \%)$ & $32(57 \%)$ & $39(55 \%)$ & 0.665 \\
Diabetes (n, \%) & $0(0 \%)$ & $11(20 \%)$ & $11(15 \%)$ & 0.143 \\
Hypercholesterolemia (n, \%) & $4(27 \%)$ & $24(43 \%)$ & $28(39 \%)$ & 0.400 \\
Any structural heart disease (n, \%) & $4(27 \%)$ & $21(38 \%)$ & $25(35 \%)$ & 0.634 \\
Valvular heart disease (n, \%) & $2(13 \%)$ & $16(29 \%)$ & $18(25 \%)$ & 0.384 \\
Hypertrophic LV (n, \%) & $5(33 \%)$ & $14(25 \%)$ & $19(27 \%)$ & 0.750 \\
Impaired LV function (n, \%) & $1(7 \%)$ & $13(23 \%)$ & $14(20 \%)$ & 0.287 \\
Previous electric cardioversion (n, \%) & $3(20 \%)$ & $29(54 \%)$ & $32(46 \%)$ & 0.057 \\
Previous AF ablation (n, \%) & $0(0 \%)$ & $4(7 \%)$ & $4(6 \%)$ & 0.663 \\
Left Atrium dilatation (n, \%) & $5(33 \%)$ & $45(80 \%)$ & $50(70 \%)$ & 0.001 \\
ACE inhibitors /ARBs (n, \%) & $1(7 \%)$ & $22(39 \%)$ & $23(32 \%)$ & 0.037 \\
Lipid lowering agents (n, \%) & $5(33 \%)$ & $18(32 \%)$ & $23(32 \%)$ & 1 \\
Betablockers (n, \%) & $6(40 \%)$ & $37(66 \%)$ & $43(61 \%)$ & 0.124 \\
Amiodarone (n, \%) & $1(7 \%)$ & $19(34 \%)$ & $20(28 \%)$ & 0.078 \\
Flecainide/Propapenone (n, \%) & $8(53 \%)$ & $11(20 \%)$ & $19(27 \%)$ & 0.022 \\
Calcium channel antagonists (n, \%) & $1(7 \%)$ & $5(9 \%)$ & $6(8 \%)$ & 1 \\
\hline
\end{tabular}

applied. Thus, we obtain a spectrum with both frequency and time informa-

tion. From the different versions of the uncertainty principle it is known that 
one cannot expect to find a time-frequency representation with perfect accuracy both in time and frequency. Hence, regarding windows width, we should take into account that choosing too narrow windows will result on poor low frequencies resolution, whereas using a too much wide window produces poor time resolution at high frequencies.

In order to address this problem, the Wavelet Transform introduces the feature known as progressive resolution, using scaled replications of a chosen mother wavelet. However, the Wavelet Transform does not use complex sinusoidal basis functions, so it measures a kind of scale information, but not frequency information directly.

The Stockwell Transform (ST) is able to provide frequency-dependent resolution by moving a scalable Gaussian window ${ }^{19,20}$. The ST of a signal $f$ is defined by

$$
(S f)(\tau, \nu)=|\nu| \int_{-\infty}^{\infty} g_{0}(\nu(t-\tau)) e^{-2 \pi i \nu t} f(t) d t
$$

where $g_{0}$ denotes the Gaussian window. This is not a wavelet transform due to the exponential term in the integral nor it is a Gabor transform due to the dilation term appearing in the window $g_{0}$.

As the size of the essential support of the window $g_{0}(\nu(\cdot-\tau))$ increases as the frequency $\nu$ becomes small, the ST provides a very good frequency resolution at low frequencies and a good temporal resolution at high frequencies. The group structure behind the ST allows the discretization ${ }^{21,22}$, meaning 
that all the information about the signal can be extracted from a convenient sample of its ST. The ST can be written in terms of the spectrum of the signal as

$$
(S f)(\tau, \nu)=e^{-2 \pi i \nu \tau} \int_{-\infty}^{\infty} e^{2 \pi i \tau \omega} e^{-2 \pi^{2}(\omega-\nu)^{2} / \nu^{2}} \widehat{f}(\omega) d \omega
$$

Combination of progressive resolution and globally referenced frequency and phase measurements with the use of sinusoidal basis functions point to the ST as a very useful tool for biomedical signal analysis. Since the ST uses a redundant sampling scheme, the Discrete Orthonormal Stockwell Transform $(\mathrm{DOST})^{23}$ proposes to reduce this redundant information by using a dyadic sampling scheme of the time-frequency domain and applying an orthonormal transform, maximizing efficiency by obtaining a representation with $N$ points from a $N$ points signal, as shown in Figure 1. It presents a computational cost of $O\left(N^{2}\right)$.

The General Fourier-family Transform (GFT) introduced by Brown et al. $(2010)^{24}$ is a general time-frequency transform that allows the use of arbitrary frequency adaptive windows combined with an efficient implementation using FFTs, which leads to a computational cost of $O(N \log N)$. So, GFT is able to produce a complex spectrum with both frequency with progressive resolution and globally referenced phase information. It also presents a dyadic sampling scheme, obtaining a vector of length $N$ from a signal with $N$ samples (Figure 1). We have also chosen a Gaussian window (as in the 


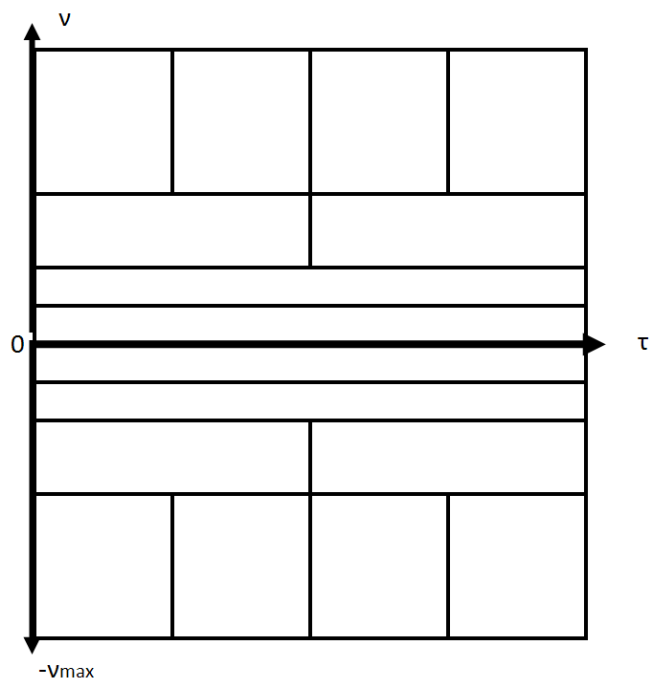

Figure 1: Example of time-frequency spectrum with a dyadic sampling scheme (with $\mathrm{N}=16$ samples) for signal length $\mathrm{N}=16$ samples. Horizontal axis represent time, whereas vertical axis represent positive $(\nu>0)$ and negative $(\nu<0)$ frequencies. Each rectangle corresponds to one GFT coefficient.

ST) for several reasons ${ }^{25}$ : the Fourier Transform of a Gaussian is a Gaussian, there are no sidelobes in a Gaussian function (so associated artifacts masking local maxima are avoided), and Gaussian window minimizes the quadratic time-frequency moment about a time-frequency sample ${ }^{26}$. Therefore, in our application we use Algorithm 1 in $^{24}$ with a Gaussian window to perform signal analysis and extract features from ECGs.

\subsection{Features}

We will analyse the GFT time-frequency transform of the bipolar lead II. Raw data is obtained from surface electrocardiograms stored with PDF format $^{27}$. Figure 2 displays the temporal patterns (after baseline and powerline 
noise removing) and their corresponding GFT transforms for two paroxysmal cases. Figure 3 displays the analogous information by using two persistent cases. It can be observed that temporal patterns are different in amplitude, morphology and non-regular cardiac rhythm. There exist differences between paroxysmal and persistent episodes by looking at their GFTs. The relevant frequency bands in our case, i.e. frequency bands where the power spectrum of the ECG is concentrated, are less than $60 \mathrm{~Hz}^{2}$. In our case, $60 \mathrm{~Hz}$ corresponds to 0.12 in figures 2 and 3 , because frequency axis is scaled to the normalized frequency (obtained by dividing them by the sampling frequency). In these figures we can note that paroxysmal segments present significant components along all the temporal axis for some of the relevant frequency bands, whereas for persistent segments significant frequency values are concentrated around QRS complexes.

So, we propose to consider the total variation of the GFT along temporal axis, for each relevant frequency band. More precisely, if $\left\{z_{1}, \ldots, z_{N}\right\}$ denotes the values along the temporal axis on a given frequency band we put $z_{j+1}-z_{j}=r_{j} \exp \left(i \varphi_{j}\right), r_{j} \geq 0$ and $-\pi \leq \varphi_{j} \leq \pi$, and consider the feature $\sum_{j=1}^{N-1} r_{j}$. In order to obtain more information, $\sum_{j=1}^{N-1}\left|\varphi_{j}\right|$ is also considered. Since each temporal segment presents intra-patient arrhythmias and differences in amplitude, we normalize each segment to the same range. Thus, we first normalize each patient to range $[0,1]$, where 0 represents the minimum amplitude and 1 represents the maximum voltage amplitude. Then, we also normalize to the number of QRS complexes present in each segment. 
To increment the information to discriminate between both subtypes of AF, we also include information about energy of the GFT transform for each frequency band along temporal axis. Figure 4 displays the observed means of the proposed features for all frequency bands of the GFT. It shows that paroxysmal subjects present smaller values of sum of differences (of modulus and phase) and smaller energy than persistent subjects for normalized frequencies from 0.003421 to 0.06207 (which correspond to frequencies from $1.71 \mathrm{~Hz}$ to $31.04 \mathrm{~Hz}$, respectively). Thus, we propose to consider the reported features as input to the classification that will be detailed in Section 3.3.
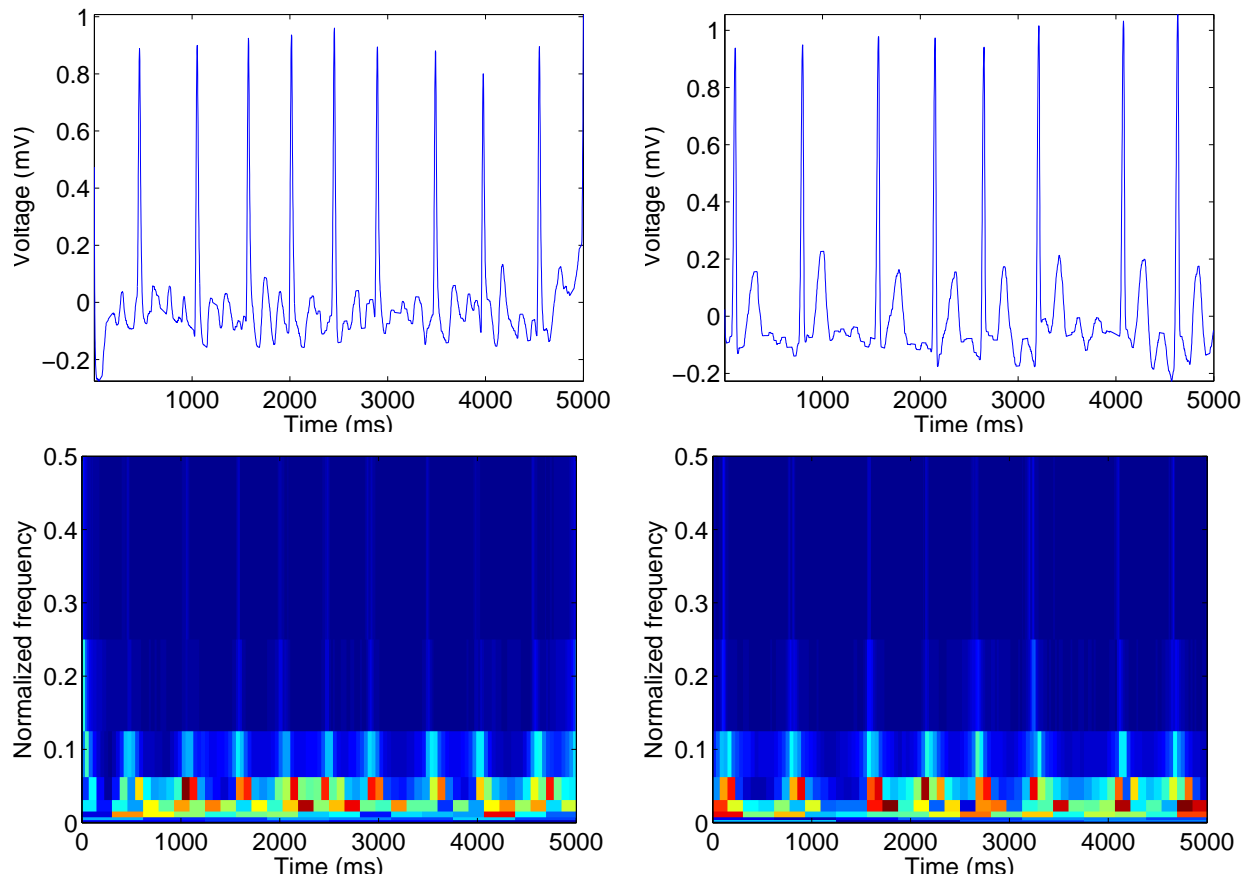

Figure 2: Examples of paroxysmal AF segments (first row) and the corresponding modulus of the associated GFT time-frequency transforms (second row). GFTs are represented using a colourmap where warm colours represent higher values than cold colours. 

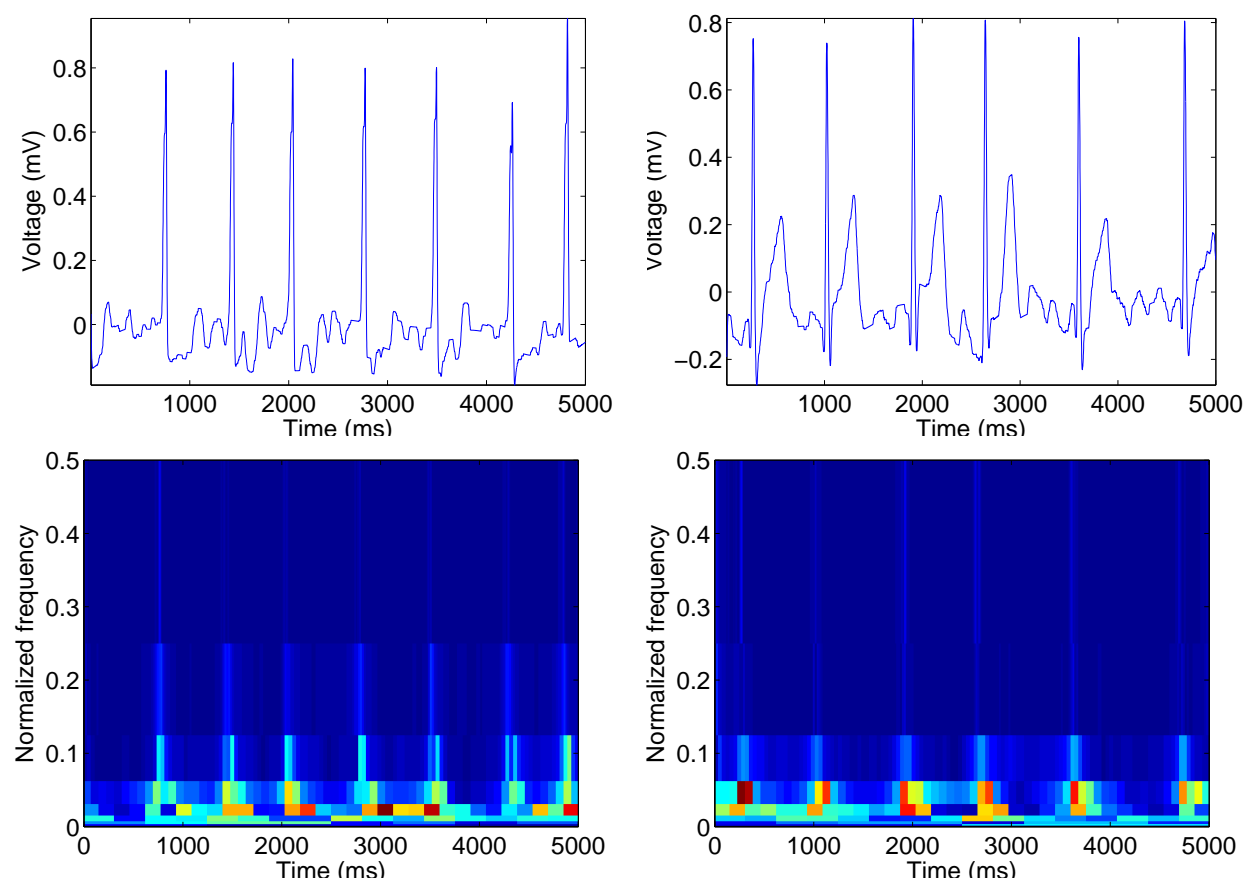

Figure 3: Examples of persistent AF segments (first row) and the corresponding modulus of the associated GFT time-frequency transforms (second row). GFTs are represented using a colourmap where warm colours represent higher values than cold colours.

\subsection{Classification}

We will consider different features extracted from the relevant frequency bands of the GFT time-frequency transform and the Support Vector Machines $(\mathrm{SVM})^{28}$ will be used to classify.

SVM classifies by finding the hyperplane that best separates all data of the training set i.e. the one that presents the largest margin between classes 29. The SVM requires to solve the following minimization problem

$$
\min _{w, b, \xi} \frac{1}{2} w^{T} w+C \sum_{i=1}^{l} \xi_{i}
$$




$$
\text { subject to } y_{i}\left(w^{T} \phi\left(x_{i}\right)+b\right) \geq 1-\xi_{i}, \quad \xi_{i} \geq 0
$$

where $x_{i} \in \mathbb{R}^{n}$ and $y_{i} \in\{1,-1\}$ are the feature vector and the classification of the i-th training observation. Here $C>0$ is the penalty parameter of the error term, whereas

$$
K\left(x_{i}, x_{j}\right)=e^{-\gamma\left\|x_{i}-x_{j}\right\|^{2}}, \gamma>0
$$

is the kernel function used. Cross-validation has been used in order to prevent overfitting. The LIBSVM library for support vector classification ${ }^{30}$ has been used.

Algorithm 1 details the proposed ECG processing sequence to extract features and classify AF patients.

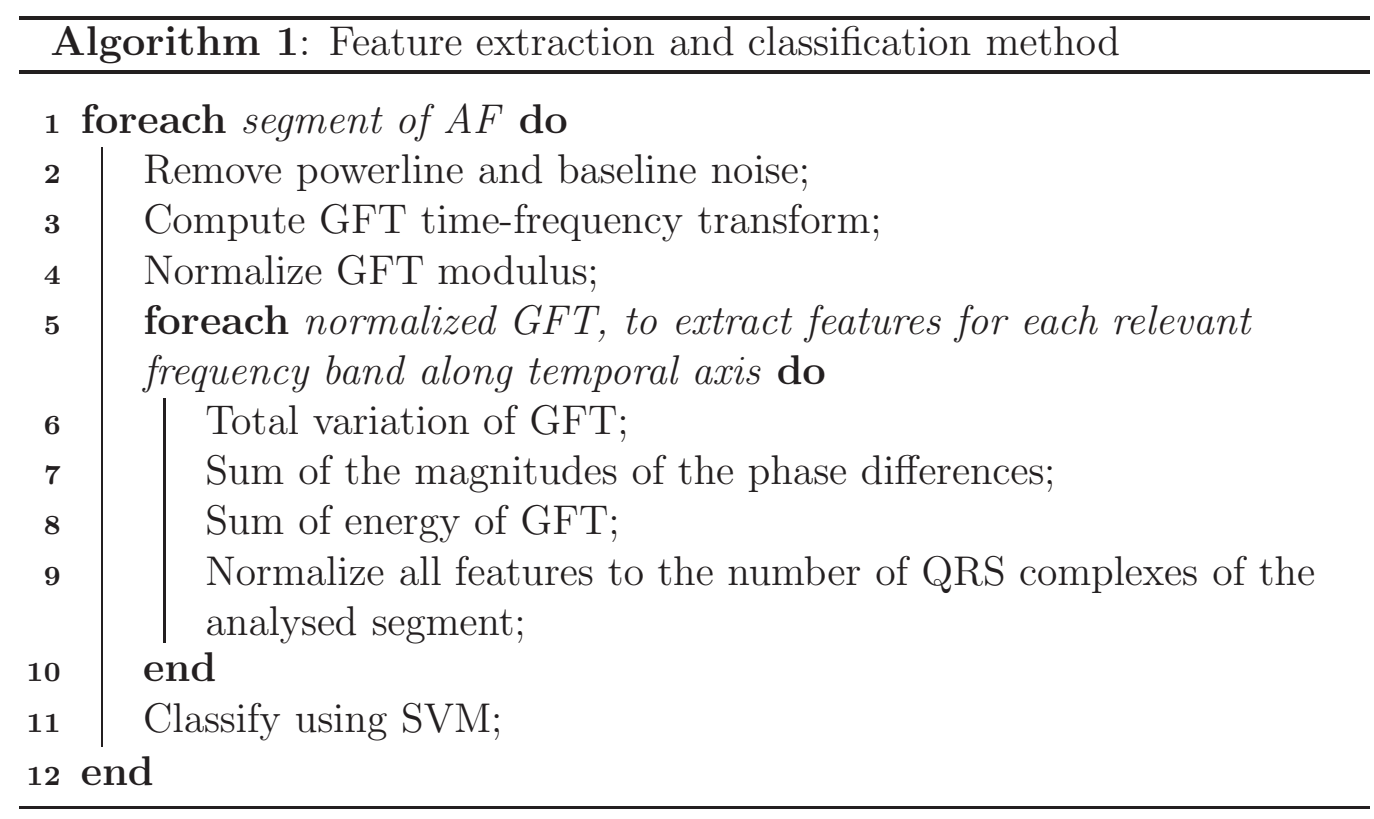




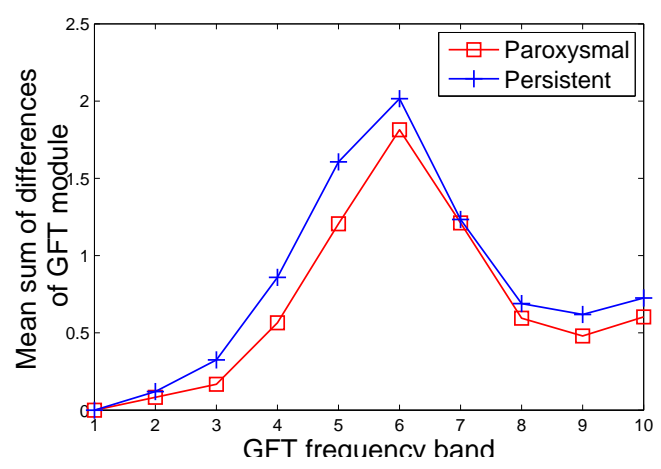

(a)

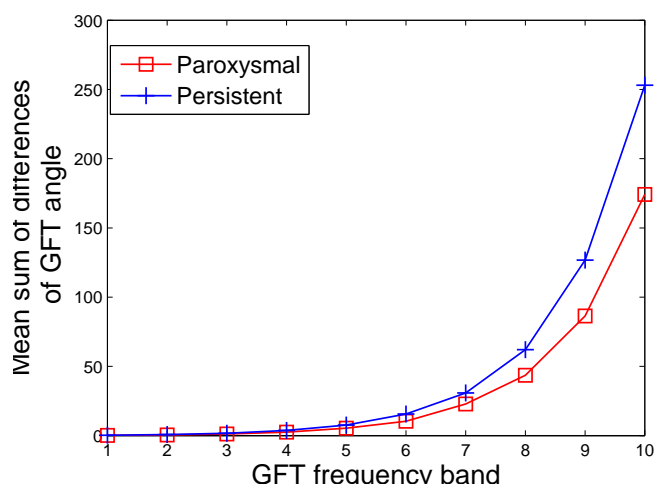

(b)

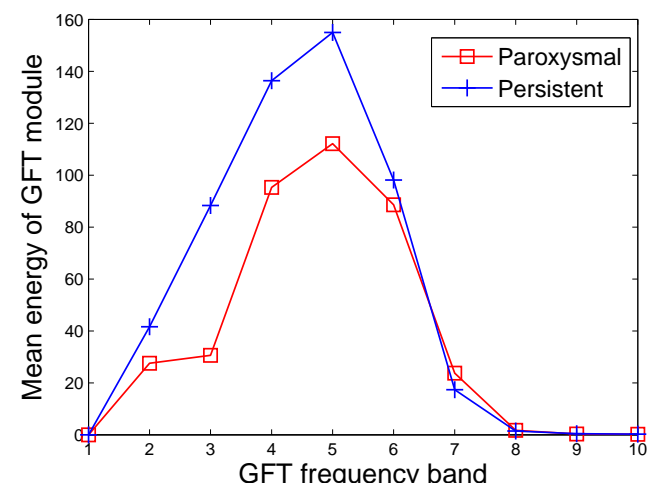

(c)

Figure 4: Features for a training set of paroxysmal and persistent subjects along each frequency band. (a) Mean of the sum of the modulus differences. (b) Mean of the sum of the phase differences. (c) Mean energy of GFT modulus. 


\section{Experimental results}

\subsection{Performance measures}

The training process of the SVM classifier has been performed in order to optimize the global accuracy (or proportion of correctly classified observations) given by

$$
A C C=\frac{T P}{T P+F P}
$$

where TP (true positive) is the number of the paroxysmal and persistent segments correctly classified while $F P$ (false positive) is the sum of the number of paroxysmal segments classified as persistent and the persistent segments classified as paroxysmal. It will be used the sensitivity (or recall) defined as the proportion of paroxysmal episodes correctly classified from the total number of paroxysmal AF episodes. The specificity is the ratio of the correctly classified persistent AF's.

Bootstrap estimators for the standard errors of the just defined measures will be proposed using 1000 resamples $^{31}$. Results are detailed in the following section.

\subsection{Results}

We have 56 persistent versus 15 paroxysmal cases, i.e. a non-balanced study. So, it will be used the bootstrap to estimate the variance of the sample means of performance measures and to calculate the most suitable number of signals for training. 
Firstly, we will give the bootstrap estimators for the mean of the performance measures. We have decided, due to the different sample sizes of signal types, to use a similar number of training samples of each subtype in order to avoid biases. It can be observed in Table 2 the influence of the number of training signals, varying from 24-18-12 (12-9-6 signals for each AF subtype, respectively) to 15 training signals (6 paroxysmal and 9 persistent). The global accuracy has its maximum with 15 training signals. The $95 \%$ bootstrap confidence intervals are shown in Table 3, showing a global accuracy of around a $75 \%$ by training with 15 randomly chosen signals.

Our dataset corresponds to an heterogeneous group suffering from other cardiac illnesses as ischemia, left bundle branch block or a heart pacemaker. Thus, for the training process, we have chosen segments of those patients that can be clinically considered as "models" for each subtype of AF without other relevant cardiac abnormalities. Results depicted in Table 4 have been obtained by using 15 training signals (6 paroxysmal and 9 persistent).

Table 2: Bootstrap estimators of sensitivity, specificity and global accuracy using 1000 simulations and different numbers of training samples.

\begin{tabular}{cccc}
\hline Number of training signals & Sensitivity & Specificity & Accuracy \\
\hline 24 & 0.7607 & 0.7486 & 0.751 \\
18 & 0.7497 & 0.7192 & 0.7253 \\
12 & 0.7487 & 0.6779 & 0.6919 \\
15 & 0.5856 & 0.8123 & 0.7676 \\
\hline
\end{tabular}

Table 4 distinguishes if the subset used for training the SVM classifier is 
Table 3: $95 \%$ bootstrap confidence intervals for mean sensitivity, specificity and global accuracy using 1000 simulations. Results are depicted for different number of training signals.

\begin{tabular}{cccc}
\hline Number of training signals & Sensitivity & Specificity & Accuracy \\
\hline 24 & {$[0.724,0.797]$} & {$[0.717,0.781]$} & {$[0.731,0.771]$} \\
18 & {$[0.709,0.791]$} & {$[0.684,0.754]$} & {$[0.703,0.748]$} \\
12 & {$[0.703,0.794]$} & {$[0.636,0.720]$} & {$[0.665,0.719]$} \\
15 & {$[0.535,0.634]$} & {$[0.783,0.841]$} & {$[0.751,0.784]$} \\
\hline
\end{tabular}

included or not when computing the performances. These results show that around an $80 \%$ of the AF segments were correctly classified, with similar sensitivity and specificity.

Table 4: Classification results: means of sensitivity, specificity and global accuracy. Classification done with SVM trained with 15 relevant signals (6 paroxysmal and 9 persistent). The whole data set (training+testing) is composed by 71 signals ( 15 paroxysmal and 56 persistent), whereas the test set is composed by 56 signals ( 9 paroxysmal and 47 persistent).

\begin{tabular}{cccc}
\hline & Sensitivity & Specificity & Accuracy \\
\hline Whole data set & 0.801 & 0.867 & 0.786 \\
Test set & 0.772 & 0.778 & 0.771 \\
\hline
\end{tabular}

\section{Discussion}

In this paper, differences between paroxysmal and persistent AF electrocardiograms have been analyzed by means of time-frequency transforms. Promising results have been obtained, specially when taking into account the population sample: a heterogeneous group (regarding AF evolution and medication). 
It is important to remark that it is more usual in the literature to consider homogenous groups of patients with similar age, under the same antiarrhythmic therapy, or in an AF episode close to end. For example, the method proposed by Alcaraz et al. (2011) ${ }^{32}$ is able to classify paroxysmal and persistent AF episodes with an accuracy higher than 95\%, but at the expense of using an homogeneous group of patients who all were under the same anticoagulant and antiarrhythmic drug therapy.

It is also important to note that, from a clinical point of view, the consequences (or costs) of the two possible misclassifications are very different and this should be taken into account. An early paroxysmal AF detection will allow an early treatment (for instance, using an ablation) and, possibly, to stop the progression to persistent AF.

In spite of our unbalanced dataset (15 paroxysmal AF patients towards 56 persistent), we obtain similar sensitivity and specificity performances. Thus, although the number of persistent segments is very much larger than the paroxysmal, classification results are not biased to persistent subtype.

Hence, paroxysmal or persistent episodes are accurately classified in a $78 \%$ and $77 \%$ of the cases, respectively. This will help electrophysiologists and clinical staff to choose the most suitable therapy in each case, revealing potential patients where an electrical cardioversion could be appropriate to prevent from the natural progression of atrial fibrillation towards a persistent or permanent state.

Finally, although no significant variations can be observed by looking at 
the surface ECG, physiological changes associated with persistent AF (such as atrial fibrosis and remodeling) compared with much healthier atrias which correspond to patients with paroxysmal AF (which are smaller, without fibrosis or remodeling) are revealed when applying time-frequency analysis. Thus, with a simple, cheap and widely available test as the ECG, we are able to differentiate clinical subtypes of AF, which could save costs, increase the effectiveness of treatments and reduce possible risks or side effects.

\section{Conclusions}

A new classification method of atrial fibrillation subtypes has been proposed based on the analysis of short electrocardiogram segments. The method uses the efficient General-Fourier family time-frequency transform to distinguish between paroxysmal and persistent episodes and analyses the spectral content of the relevant frequency bands along temporal axis for feature extraction. Then, segments are classified using a SVM trained to maximize global accuracy. Good experimental results on real ECG records of atrial fibrillation episodes have been achieved specially when taking into account the heterogeneous dataset used (regarding recurrence of episodes, and pharmacological or surgical antiarrhythmic treatment). Future developments will focus on improving performance in a more heterogeneous enlarged data set, in addition to studying the possible recurrence and the progressive evolution of the arrhythmia. 


\title{
Acknowledgements
}

This work was supported by grants MTM2010-15200, PrometeoII/2013/013 and UPV-IIS La Fe, 2012/0468.

\section{Competing interests}

\author{
None declared
}

\section{Ethical approval}

Ethical approval was obtained by the participating centres: Hospital Universitari i Politècnic La Fe and Instituto de Investigación Sanitaria La Fe. Patients also signed an agreement allowing to use their data for clinical studies.

\section{References}

1. Wann L, Curtis A, January C, Ellenbogen K, Lowe J, Estes N, et al. 2011 accf/aha/hrs focused update on the management of patients with atrial fibrillation (updating the 2006 guideline): a report of the american college of cardiology foundation/american heart association task force on practice guidelines. Circulation 2011;123(1):104-123.

2. Sörnmo L, Laguna P. Bioelectrical Signal Processing in Cardiac and Neurological Applications. Elsevier Academic Press; 2005. 
3. Fuster V, Rydén L, Cannom D, Crijns H, Curtis A, Ellenbogen K, et al. Acc/aha/esc 2006 guidelines for the management of patients with atrial fibrillation: a report of the american college of cardiology/american heart association task force on practice guidelines and the european society of cardiology committee for practice guidelines (writing committee to revise the 2001 guidelines for the management of patients with atrial fibrillation): developed in collaboration with the european heart rhythm association and the heart rhythm society. Circulation 2006; 114(7):e257-e354.

4. Markides V, Schilling R. Atrial fibrillation: classification, pathophysiology, mechanisms and drug treatment. Heart 2003;89(8):939-943.

5. Chiarugi F. New developments in the automatic analysis of the surface ecg: The case of atrial fibrillation. Hellenic Journal of Cardiology 2008; 49(4):207-221.

6. Bollmann A, Husser D, Mainardi L, Lombardi F, Langley P, Murray A, et al. Analysis of surface electrocardiograms in atrial fibrillation: techniques, research, and clinical applications. Europace 2006;8(11):911-926.

7. Martinez A, Alcaraz R, Rieta J. Study on the p-wave feature time course as early predictors of paroxysmal atrial fibrillation. Physiological Measurement 2012;33(12):1959-1974.

8. Kotstka P, Tkacz J. Feature extraction in time-frequency signal analysis 
by means of matched wavelets as a feature generator. Conf Proc IEEE Eng Med Biol Soc.; 2011, p. 4996-4999.

9. Bukkapatnam S, Komanduri R, Yang H, Rao P, Lihand W, Malshe M, et al. Classification of atrial fibrillation episodes from sparse electrocardiogram data. Journal of Electrocardiology 2008;41(4):292-299.

10. Moody G. Spontaneous termination of atrial fibrillation: A challenge from physionet and computers in cardiology. Computers in Cardiology 2004;31:101-104.

11. Nilsson F, Stridh M, Bollmann A, Sörnmo L. Predicting spontaneous termination of atrial fibrillation using the surface ecg. Medical Engineering $\&$ Physics 2006;28(8):802-808.

12. Sandberg F, Stridh M, Sörnmo L. Frequency tracking of atrial fibrillation using hidden markov models. IEEE Transactions on Biomedical Engineering 2008;55(2):502-511.

13. Alcaraz R, Rieta J. Surface ecg organization analysis to predict paroxysmal atrial fibrillation termination. Computers in Biology and Medicine 2009;39(8):697-706.

14. Alcaraz R, Sandberg F, Sörnmo L, Rieta J. Application of frequency and sample entropy to discriminate long-term recordings of paroxysmal and persistent atrial fibrillation. Conf Proc IEEE Eng Med Biol Soc.; 2010, p. 4558-4561. 
15. Sun R, Wang Y. Predicting termination of atrial fibrillation based on the structure and quantification of the recurrence plot. Medical Engineering E Physics 2008;30(9):1105-1111.

16. Valenzuela O, Rojas I, Rojas F, Pomares H, Bernier J, Herrera J, et al. Intelligent system based on genetic programming for atrial fibrillation classification. Applied Artificial Intelligence 2009;23(10):895-909.

17. Petrutiu S, Sahakian A, Swiryn S. Abrupt changes in fibrilatory wave characteristics at the termination of paroxysmal atrial fibrillation in humans. Europace 2007;9(7):466-470.

18. Sahoo S, Lu W, Teddy S, Kim D, Feng M. Detection of atrial fibrillation from non-episodic ecg data: a review of methods. Conf Proc IEEE Eng Med Biol Soc.; 2011, p. 4992-4995.

19. Stockwell R, Mansinha L, R.P.Lowe . Localization of the complex spectrum: The S transform. IEEE Transactions of Signal Processing 1996; 44(4):998-1001.

20. Wong MW, Zhu H. A characterization of Stockwell spectra. Modern trends in pseudo-differential operators 2007;172:251-257.

21. Boggiatto P, Fernández C, Galbis A. A group representation related to the Stockwell transform. Indiana Univ Math J 2009;58(5):2277-2296.

22. Führ H, Gröchenig K. Sampling theorems on locally compact groups from oscillation estimates. Math Z 2007;255(1):177-194. 
23. Stockwell R. A basis for efficient representation of the s-transform. Digital Signal Processing 2007;17(1):371-393.

24. Brown R, Lauzon M, Frayne R. A general description of linear timefrequency transforms and formulation of a fast, invertible transform that samples the continuous S-transform spectrum nonredundantly. IEEE Transactions of Signal Processing 2010;58(1):281-290.

25. Stockwell RG. Why use the S-transform? In: Pseudo-Differential Operators: Partial Differential Equations and Time-Frequency Analysis; vol. 52 of Fields Institute Communications. AMS; 2007, p. 279-309.

26. Janssen A. Optimality property of the gaussian window spectrogram. IEEE Transactions on Signal Processing 1991;39(1):202-204.

27. Ortigosa N, Giménez V. Raw data extraction from electrocardiogramswith portable document format. Computer Methods and Programs in Biomedicine 2014;113:284-289.

28. Duda R, Hart P, Stork D. Pattern Classification. Wiley Interscience; 2000.

29. Cortes C, Vapnik V. Support-vector networks. Machine Learning 1995; $\mathbf{2 0}(3): 273-297$.

30. Chang C, Lin C. LIBSVM: A library for support vector machines. ACM Transactions on Intelligent Systems and Technology 2011;2(3):27:1$27: 27$ 
31. Efron B. Bootstrap methods: another look at the jackknife. The Annals of Statistics 1979;7(1):1-26.

32. Alcaraz R, Sandberg F, Sörnmo L, Rieta J. Classification of paroxysmal and persistent atrial fibrillation in ambulatory ecg recordings. IEEE Transactions on Biomedical Engineering 2011;58(5):1441-1449. 